\title{
Experimental Model Tests \\ of a Permanent Magnet Stirrer \\ for Aluminium Furnaces
}

\author{
Andris Bojarevics*, Toms Beinerts, \\ Martins Sarma and Yury M.Gelfgat \\ Institute of Physics University of Latvia \\ 32 Mira Str., Salaspils, LV-2169, Latvia
}

Received 14.02.2015, received in revised form 23.03.2015, accepted 30.04.2015

\begin{abstract}
A model experimental set-up was built aiming to ensure achievable similitude to large scale metal melting furnaces, where electromagnetic stirring is required to reduce the melting time and to achieve thermal and compositional uniformity. The widely used three-phase AC current linear travelling magnetic field inductor is substituted by a new energy-saving concept of a PermanentMagnet (PM) inductor, consisting of a multitude of cylindrical dipoles, which form a Halbach array and are rotated synchronously. The stirrer creates a very unstable time-dependent flow pattern in the liquid metal pool. The turbulent local velocity was measured, delivering experimental data about time-averaged flow and turbulence intensity as well. Spatial components of velocity were measured by ultrasound Doppler anemometry and by a potential difference probe with an incorporated permanent magnet, delivering mutually verifying and complementary data with higher reliability. The experimental data might be used to validate the full three-dimensional numerical simulations of the stirring produced in actual large scale metal melting furnaces.
\end{abstract}

Keywords: electromagnetic stirring, permanent magnets, travelling magnetic field, melt hydrodynamics under the field action.

DOI: $10.17516 / 1999-494 X-2015-8-5-569-575$.

(c) Siberian Federal University. All rights reserved

* Corresponding author E-mail address: andrisb@sal.lv 


\title{
Экспериментальные исследования модели
}

МГД-перемешивателя с постоянными магнитами

\section{для алюминиевых печей}

\author{
А. Бояревич, Т. Бейнертс, \\ М. Сарма, Ю.М. Гельфгат
}

Институт физики Латвийского университета Латвия, LV-2169, Саласпилс, ул. Мира, 32

Статья посвящена физическому моделированию электромагнитных и гидродинамических процессов в жидком алюминии при воздействии на расплав МГД-перемешивателя с постоянными магнитами. Экспериментальная установка была выполнена с возможностью моделирования параметров, соответствующих реальным печам, в которых электромагнитное перемешивание необходимо для уменьшения времени плавки и достижения однородного состава и распределения температуры в расплаве алюминия. В отличие от используемых в настоящее время МГД-перемешивателей с трехфазным индуктором переменного тока в статье представлен новый энергосберегающий вариант создания бегущего магнитного поля с помощью индуктора с системой синхронно вращцаюцихя ицлиндрических магнитных диполей, образуюших систему Наlbach. Такой перемешиватель создает в жидком металле потоки с высоким уровнем нестационарных пульсирующих скоростей. Проведень измерения осредненных и пульсационных характеристик возникающих турбулентных течений $и$ их взаимосвязь при различных режимах работь перемешивателя. Измерения всех трех компонент скорости проведены с помощью ультразвукового доплеровского анемометра и кондукционного анемометра со встроенным миниатюрным постоянным магнитом. Сравнение данных, полученных двумя способами, позволило с высокой надежностью оценить количественные характеристики значений скорости и использовать результаты экспериментов для анализа данных трехмерных численных расчетов перемешивающих потоков в крупномасштабньх плавильных печах.

Ключевые слова: электромагнитное перемешивание, постоянные магниты, бегущее магнитное поле, гидродинамика расплава под воздействием поля.

\section{Введение}

Электромагнитные перемешиватели (ЭМП) жидких металлов широко используются при плавке и модифицировании сплавов легких металлов в больших печах емкостью до 70 м $^{3}$ для получения однородной температуры и состава расплава. Большинство из доступных сегодня ЭМП представляют собой индукторы переменного тока, генерирующие бегущее магнитное поле. Так как безопасность, экономия энергии и продолжительность работы таких печей требуют, чтобы перемешивание осуществлялось через толстые огнеупорные стенки печи, обычно окруженные дополнительным стальным корпусом толщиной до 50 мм, бегущее магнитное поле для перемешивания расплава создается с помощью высокоамперной трехфазной обмотки в виде медных катушек большого размера, подключенных к сети трехфазного тока. Для интенсивного перемешивания расплава в больших емкостях частота переменного тока не должна превышать 0,2-1 Гц, что позволяет увеличить глубину проникновения электромагнитного воздействия, ограниченного скин-эффектом $[1,2]$. Наиболее широко используемые сегодня электромагнитные перемешиватели переменного тока 
энергозатратны и требуют интенсивного водяного или воздушного охлаждения катушек индуктора.

Альтернативой для перемешивателей переменного тока, создающих бегущее магнитное поле, являются перемешиватели с постоянными магнитами (ППМ). Они, как правило, имеют систему расположенных аксиально-симметрично по окружности постоянных магнитов переменной полярности, которые при вращении образуют бегущее магнитное поле в жидком металле. Для проникновения электромагнитного воздействия через толстые стенки печи постоянные магниты должны быть относительно большого размера. Поэтому желательно также уменьшать толщину стенки или изменить ее конфигурацию [3-5].

Предлагаем новую концепцию ППМ [6-8] и обсудим результаты модельных экспериментов, посвященных изучению эффективности конструкции для перемешивания алюминия в крупногабаритных печах.

\section{Экспериментальная модель и критерии подобия}

Предлагаемый электромагнитный перемешиватель с постоянными магнитами (ППМ), создающий линейное бегущее магнитное поле, состоит из системы параллельно расположенных цилиндрических постоянных магнитных диполей с направлением вектора намагниченности каждого цилиндра перпендикулярно его оси. Магнитные цилиндры механически связаны друг с другом, что позволяет всей системе синхронно вращаться, сохраняя при этом постоянный неизменный сдвиг фаз направления вектора намагниченности между цилиндрами. Указанное расположение постоянных магнитов образует систему Халбаха (Halbach), обеспечивающую более высокое значение индукции магнитного поля в жидком металле вблизи стенки емкости с расплавом [5].

При синхронном вращении цилиндров бегущее магнитное поле, направленное параллельно касательной вращающихся магнитов, проникает в зону с жидким металлом [6]. Схема модельного эксперимента представлена на рис. 1. Геометрическое подобие экспериментальной модели предлагаемому крупномасштабному перемешивателю для алюминия задано системой безразмерных геометрических соотношений: $\mathrm{h} / \tau=0,25 ; \mathrm{H} / \tau=0,6 ; \mathrm{W} / \tau=0,93 ; \mathrm{L} / \tau=2,7$; $\mathrm{W}_{\mathrm{m}} / \tau=1,9$, где $\mathrm{H}$ - расстояние от магнитов до дна емкости с жидким металлом, $\mathrm{W}$ - длина магнитных цилиндров, $\mathrm{R}$ - радиус магнитов, $\mathrm{h}$ - толщина слоя металла, $\tau$ - полюсный шаг и $\delta=(2 / \sigma \omega \mu)^{-1}-$ глубина скин-слоя в жидком металле при угловой частоте $\omega$ вращения магни-
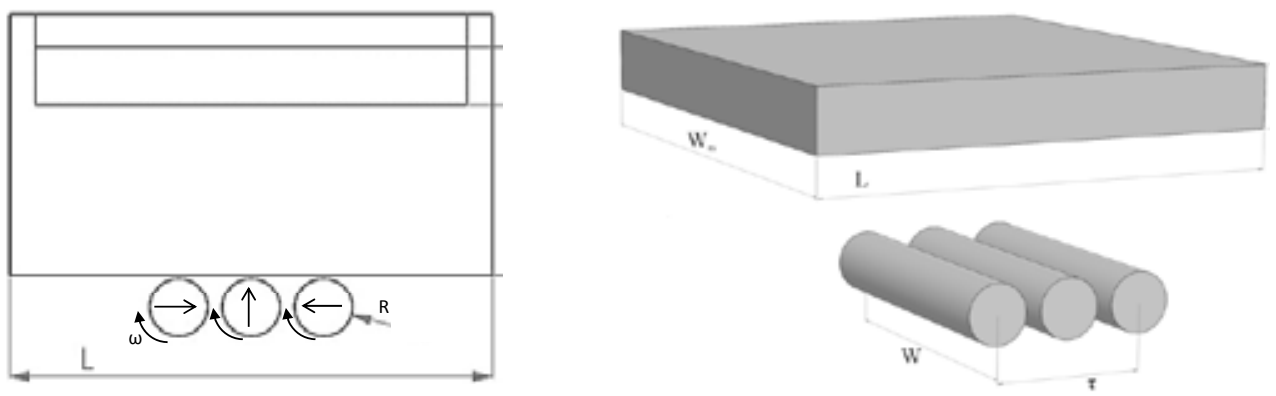

Рис. 1. Схема экспериментальной установки 
тов. Физическое подобие модели и реальной печи требует идентичности следующих безразмерных критериев:

$$
\Omega_{d}=\left(\frac{\tau}{\delta}\right)^{2} \text { и } N=\frac{B}{\omega \tau \sqrt{\mu \rho}}
$$

где $\rho$ - плотность расплава; $\mu$ - магнитная проницаемость; В - величина индукции магнитного поля в зоне жидкого металла; $\Omega_{\mathrm{d}}$ - безразмерная частота и $N$ - параметр электромагнитного взаимодействия.

Цель модельного эксперимента - оценить ожидаемые характеристики перемешивающих течений жидкого металла в крупногабаритной емкости с алюминием, в 7,5 раза превышающих размеры модели. Поскольку идентичность обоих критериев физического подобия не может быть соблюдена на одной модели, приоритетным было выбрано значение безразмерной частоты, поскольку толщина скин-слоя не должна превышать глубину металла в емкости с расплавом. Следует отметить, что параметр электромагнитного взаимодействия как для модельного эксперимента, так и для предлагаемой большой печи с расплавом алюминия был бы гораздо меньше единицы, $\mathrm{N}<<1$. Однако в модельном эксперименте он был на порядок величины меньше, чем для крупногабаритной емкости с расплавом алюминия. Условие $N=\mathrm{idem}$ (одновременно с $\Omega_{\mathrm{d}}=\mathrm{idem}$ ) не может быть достигнуто из-за физических ограничений, определяемых величиной остаточной намагниченности материала постоянного магнита. Предполагается проведение второго модельного эксперимента для проверки подобия $\mathrm{N}$ без соблюдения условия $\Omega_{\mathrm{d}}=$ idem.

В эксперименте в качестве модельного расплава применяли эвтектический сплав InGaSn, плотность которого $\rho=6400$ кг $/ \mathrm{M}^{3}$, электропроводность $\sigma=3,3 \cdot 10^{6} \mathrm{~cm} / \mathrm{M}$ и кинематическая вязкость $v=3,1 \cdot 10^{7} \mathrm{~m}^{2} / \mathrm{c}$. Для расплава алюминия использовались характеристики чистого алюминия.

Скорость жидкого металла измеряли двумя способами - кондукционным анемометром со встроенным миниатюрным постоянным магнитом и ультразвуковым доплеровским анемометром, позволяющими сравнить полученные результаты.

\section{Результаты эксперимента}

Локальная скорость течения расплава измерялась в двух вертикальных сечениях, перпендикулярных направлению бегущего магнитного поля. При достаточно большой дистанции между цилиндрическими постоянными магнитами и слоем жидкого металла $\mathrm{H} / \tau=0,6$, или равнялось 2,5 диаметра цилиндров. При этом амплитуда индукции бегущего магнитного поля не превышала 10 мТ. При скорости вращения цилиндров 50 об/с величина скорости течения в расплаве над перемешивателем превышала $10 \mathrm{~cm} / \mathrm{c}$. Основное назначение перемешивателя - достижение высокого интегрального расхода во всем объеме емкости с металлом. Для оценки интенсивности подобного перемешивания на рис. 2 и 3 приведены экспериментально полученные значения осредненных и пульсационных скоростей в потоках расплава. На рис. 2 замеры проведены в вертикальном сечении над продольной средней точкой перемешивателя. Здесь расход составлял 0,47 л/с. На рис. 3 замеры проведены в сечении, расположенном вниз по течению у отметки $0,2 \mathrm{~L}$ от торцевой стенки. Здесь расход составлял 0,14 л/с. При пересчете указанных 


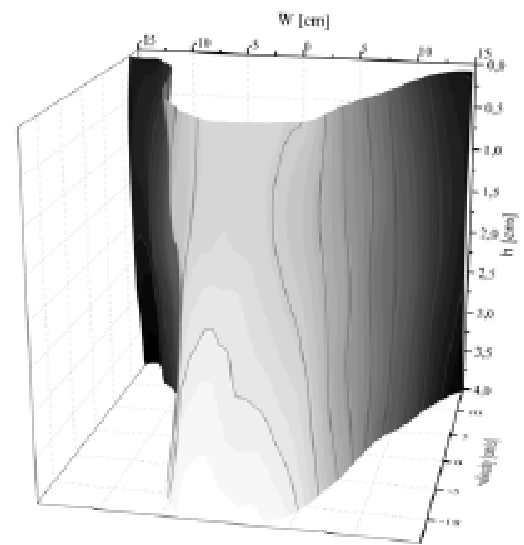

a
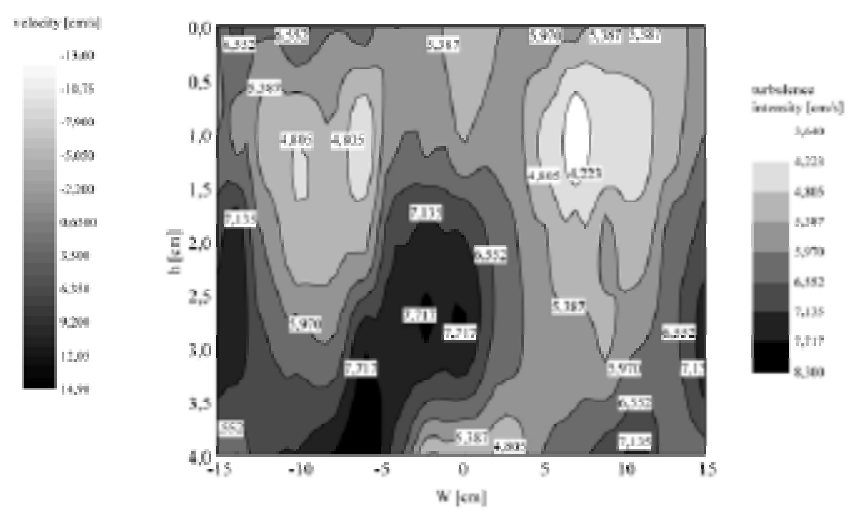

6

Рис. 2. Поле скоростей над средним цилиндрическим магнитом (а) и турбулентные пульсации скорости в этом сечении (б)

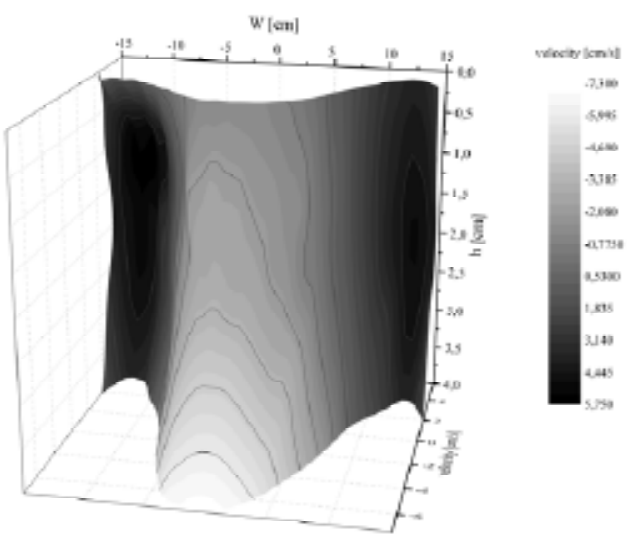

a

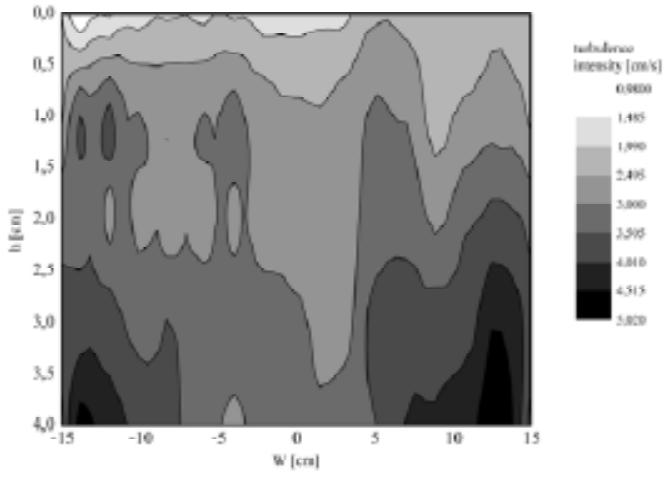

6

Рис. 3. Поле скоростей между последним цилиндрическим магнитом и стенкой емкости (а) и турбулентные пульсации скорости в этом сечении (б)

данных для емкости с расплавом алюминия, в 7,5 раза превышающей экспериментальную модель, расход составил бы 26 л/с, или 220 т/ч, при скорости вращения цилиндров постоянных магнитов 0,3 об/с.

Для оценки качества перемешивания во всем объеме печи дополнительно было изучено течение расплава в очень неоднозначной области - в угловой зоне емкости с расплавом. Исходя из общих представлений, осредненная по времени скорость течения расплава в этой области емкости должна быть небольшой, стремящейся к нулю. Однако рис. 4 свидетельствует об очень интенсивном перемешивании даже в этой области емкости, что обусловлено крайне неустойчивым характером течений в расплаве. Интенсивность турбулентных пульсаций во 


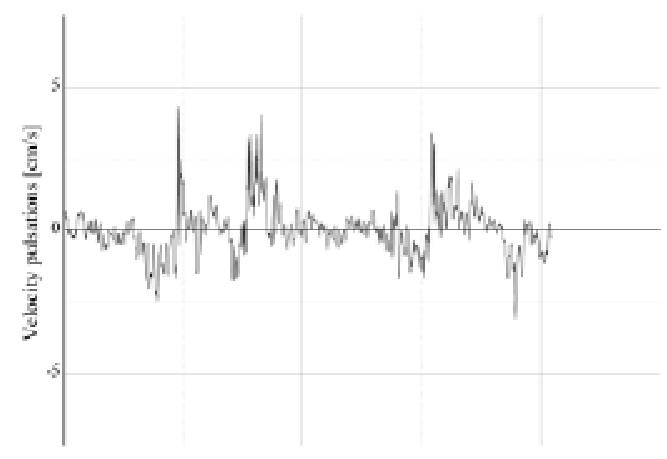

a

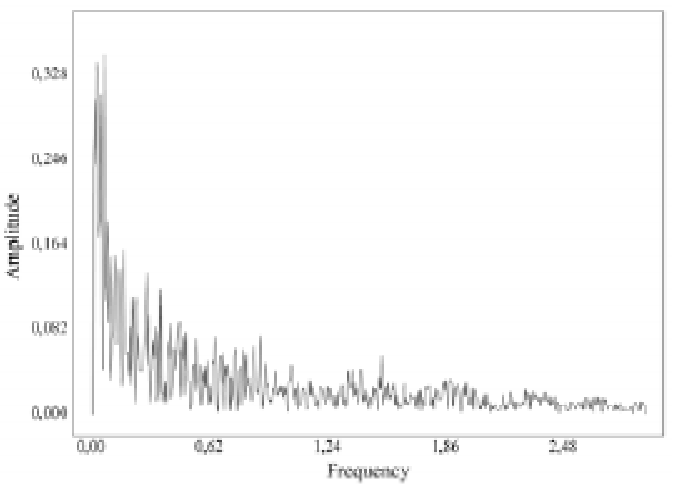

6

Рис. 4. Зависимость осредненной скорости от времени (а) и спектр Фурье в угловой зоне емкости (на расстоянии 1 см от обеих стенок и под поверхностью расплава) (б). Средняя скорость течения 0,9 см/с. Амплитуда пульсаций скорости $7,41 \mathrm{~cm} / \mathrm{c}$

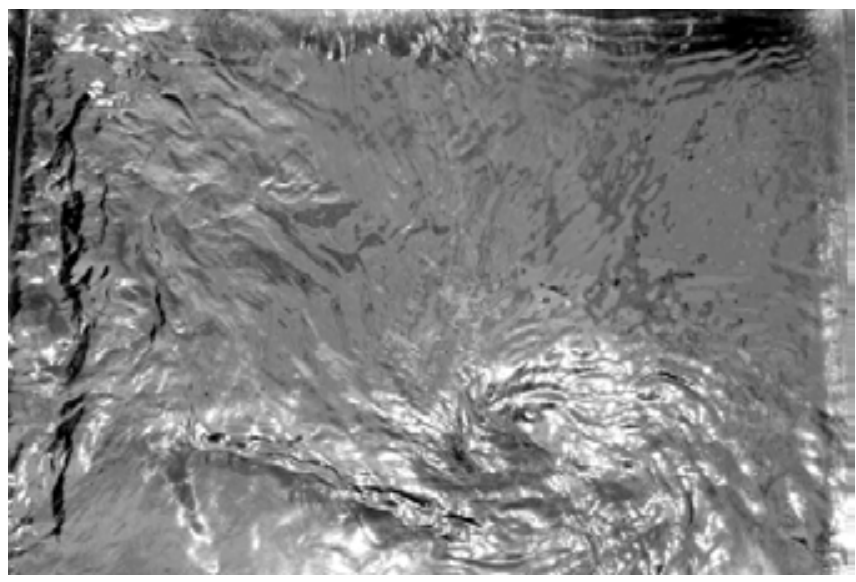

Рис. 5. Фотография вихря в расплаве InGaSn, когда перемешивание происходит при высокой угловой частоте

всем объеме расплава здесь достаточно высокая. Максимальные амплитуды флуктуаций скорости в угловой области почти такие же, как максимальное значение осредненной по времени скорости струи на дне емкости непосредственно над центральной областью перемешивателя. Спектры турбулентных пульсаций скорости показаны справа на рис. 4; они свидетельствуют, что крупномасштабные вихревые потоки, соответствующие очень низким частотам, доминируют при перемешивании расплава. Очень высокая неустойчивость вышеуказанных течений иллюстрируется фотографией на рис. 5, где видны значительные деформации свободной поверхности расплава.

\section{Заключение}

Исследованная модель МГД-перемешивателя с цилиндрическими постоянными магнитами не полностью подобна большим промышленным печам для плавки и перемешивания 
алюминия, поскольку нельзя было получить полного подобия соответствующих физических безразмерных критериев. Тем не менее, так как оба исследуемых варианта включают параметр электромагнитного взаимодействия гораздо меньше единицы $(\mathrm{N}<<1)$, полученный результат можно считать достаточно убедительным для доказательства возможности применения предлагаемого линейного индуктора с постоянными цилиндрическими магнитами для перемешивания алюминия в печах с объемом емкости для расплава до 50 т и толщиной дна емкости до 50 см. Энергопотребление такого перемешивателя не превышает 10-15 кВт, и нет необходимости в водяном или интенсивном воздушном охлаждении. Данные модельных экспериментов будут также использованы для проверки результатов численных расчетов трехмерной модели перемешивателя, находящейся в настоящее время в стадии разработки.

Участие А. Бояревича и М. Сармы в данном исследовании было профинансировано Европейским социальным фондом (проект № 2013/0018/1DP/1.1.1.2.0/13/ APIA/VIAA/061).

\section{Список литературы}

[1] Savenas M., Sjoden O. // J. Aluminium Today, June 2001. P. 910.

[2] Тимофеев В.Н., Христинич Р.М., Бояков С.Я. и др. // Цветные металлы Сибири - 2009: Сб. докладов I Международного конгресса. Красноярск, 2009. С. 657.

[3] Калнинь Т.К. Явнополюсные МГД-насосы. Рига: Зинатне, 1969. 171.

[4] Takahashi K. Pat.: No: CN 101837433(A). Japan / public. 29.05.2009.

[5] Bucenieks I. // The $14^{\text {th }}$ International Conference on Nuclear Engineering. Miami, Florida, USA, July 2006. P. 251.

[6] Bojarevičs A., Gelfgats J., Beinerts T., Bucenieks I. Pat.: No LV 14404 B. Latvia / 2011. P. 1453-1454 (in Latvian).

[7] Bojarevičs A., Beinerts T. // Magnetohydrodynamics. 2010. Vol. 46. N 4. P. 339.

[8] Beinerts T., Bojarevičs A., Gelfgats J., Bucenieks I. // EPM 2012 Conference. China. October 2012. 